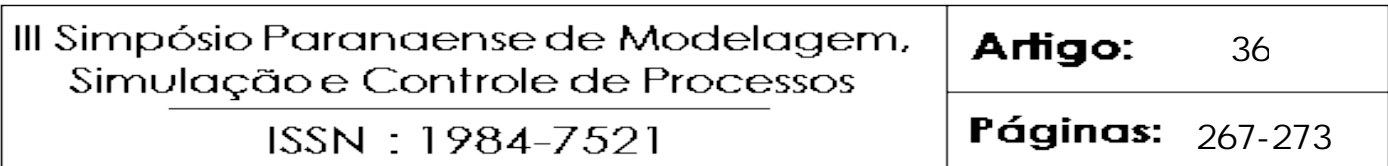

\title{
MODELAGEM E SIMULAÇÃo NO PROCESSO DE REFINO DE ÓlEO DE SOJA NA ETAPA DE NEUTRALIZAÇÃo
}

\section{Fernando Castro Vieira Filho1*, Claudia Aline Sírio Pereira1, Henryck Cesar Massao Hungaro Yoshi1, Cid Marcos Gonçalves Andrade1 e Oswaldo Curty da Motta Lima1}

1 - Universidade Estadual de Maringá - UEM - PR

(epfernando.filho@gmail.com)

Resumo - A produção de óleos vegetais, principalmente o de soja, tem função importante no setor do agronegócio mundial, sendo o Brasil um dos maiores produtores. Considerando a alta competitividade do óleo de soja, faz-se necessário uma constante busca por obter um óleo de qualidade com baixo custo operacional. No processo de refino de óleo vegetal a etapa de neutralização, a principal do ponto de vista econômico, garante a separação da borra e do óleo neutralizado. Este trabalho tem por objetivo a modelagem e simulação desta etapa na busca de gerar informações que permita a adequação dos parâmetros operacionais, afim de reduzir os custos operacionais, com a otimização da utilização dos recursos e minimizar as perdas de óleo arrastado na borra. Na planta industrial em análise, este subproduto, extraído por centrifugação, gera um arraste considerável de óleo. Deste modo, uma modelagem e simulação da etapa de neutralização proporcionou uma avaliação do processo com a utilização da simulação no software Aspen Plus®, para identificar possíveis condições ótimas de separação, fundamentada por um modelo, que neste caso foi a utilização do método termodinâmico do NRTL-RK. O modelo desenvolvido representou bem o processo da neutralização. Porém, para melhores resultados podem ser obtidos aumentando os pontos de análises de separação em diferentes temperaturas, formando um conjunto de dados $\left(T, \mathrm{~K}_{\mathrm{LL}}\right)$.

Palavra-chaves: Refino de Óleo, Neutralização, Simulação e Modelagem.

Introdução

O refino do óleo de soja agrega valor ao produto e os processos envolvidos necessitam operar nas melhores condições, a fim de propiciar a redução de custos e a melhoria da qualidade do produto final. Neste sentido, faz-se necessários o conhecimento a fundo dos processos que envolve as etapas de refino.

Os processos tecnológicos que compõe o refino se dividem em quatro grandes etapas: degomagem, neutralização, branqueamento e desodorização. Os subprodutos gerados nestes processos são: as gomas oriundas da degomagem, as borras resultadas da neutralização e o condensado proveniente da desodorização (FRÉ, 2009).

Uma das operações mais importantes do refino do óleo de soja é a neutralização. $\mathrm{Na}$ Figura 1, estão representadas de forma ilustrativa esta etapa. Nesta operação realiza-se a dosagem de ácido fosfórico e soda cáustica ao óleo de soja degomado, para neutralizar os ácidos graxos livres que não foram removidos na operação de degomagem. Além dos ácidos graxos livres removidos, também ocorre à eliminação dos fosfatídeos não hidratáveis, dos pigmentos e outras impurezas solúveis e insolúveis (ANDERSON, 1996). Assim, refere-se à neutralização como uma desacidificação, refino álcali ou cáustico (HAMMOND et al., 2005).

22 e 23 de março de 2018

Curitiba - Paraná 


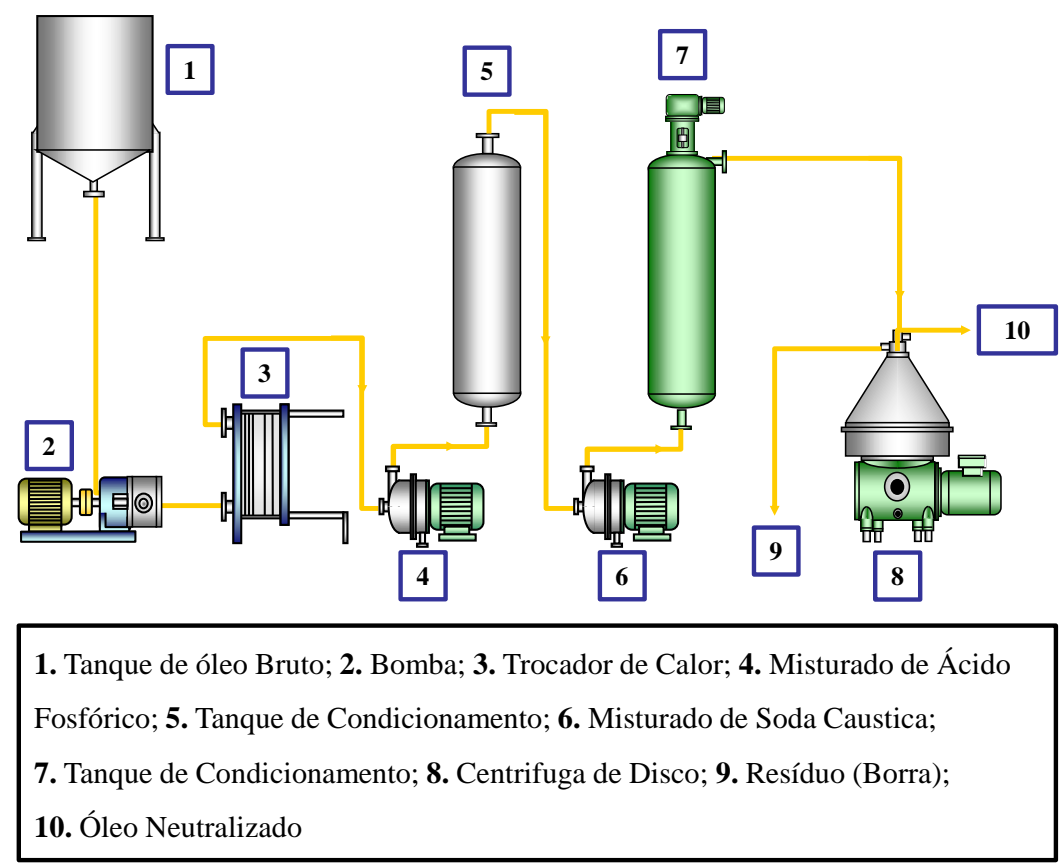

Figura 1 - Fluxo Ilustrado: Processo de Neutralização Contínua

A reação dos ácidos graxos livres com a soda leva à precipitação de oleatos de sódio (sabões). A saída do reator passa então por duas centrífugas, que promovem a separação do chamado óleo neutralizado (fase leve) e da borra (sabões) que é classificada com a fase pesada. Ao óleo neutralizado é novamente adicionado água, para remover resquícios de sabão e soda que não foram retirados nas primeiras centrífugas - esta etapa chamada de lavagem. O óleo neutralizado lavado é então encaminhado a um secador (para remover a umidade adquirida) e a água de lavagem é decantada para recuperação de uma fração de óleo que é arrastada.

\section{Modelagem da etapa de neutralização}

Para modelagem e simulação da etapa de neutralização, procurou-se fazer uma avaliação do processo por meio de simulação no software Aspen Plus® para identificar possíveis condições ótimas de separação. Contudo, devido à natureza do processo e dos componentes químicos envolvidos, isto se mostrou uma tarefa complexa e dependente de grande volume de referenciais teóricos. Trabalhos semelhantes foram desenvolvidos por Landucci (2013), Granjo (2015) e De Pretto (2017).

O primeiro passo foi identificar os componentes envolvidos no processo e a definição de uma partícula de óleo de soja. Para efeitos de simplificação, componentes semelhantes (exemplo: triglicerídeos presentes no óleo de soja) foram inseridos na forma de um único elemento representativo. Além disso, foram trabalhados apenas os principais componentes de interesse na etapa de neutralização, desprezando outros presentes em quantidades pequenas ou não fundamentais para estas etapas (exemplo: clorofila, traços de metais, e outros). A relação destes componentes está apresentada na tabela 1. 
Tabela 1 - Componentes empregados na simulação da neutralização.

\begin{tabular}{|c|c|c|c|}
\hline Componente & Identificação & Elemento Representativo & Definição \\
\hline Triglicerídeos & TGA & Trioleína (C57H104O6) & Aspen \\
\hline Umidade & WATER & Água $(\mathrm{H} 2 \mathrm{O})$ & Aspen \\
\hline Ácidos Graxos Livres & FFA & Ácido Oleico (C18H34O2) & Aspen \\
\hline Sabões & SOAPS & Oleato de Sódio (C18H33NaO2) & Aspen \\
\hline Fosfatídeos Hidratáveis & HP & (1,2-dilinoleoyl)-3-lecithin (C43H78NO7P) & User-defined \\
\hline Fosfatídeos Não-Hidratáveis & NHP & DLPE-linoleoyl (C41H74NO7P) & User-defined \\
\hline Ácido Fosfórico & $\mathrm{H} 3 \mathrm{PO} 4$ & Ácido Ortofosfórico (H3PO4) & Aspen \\
\hline Soda & $\mathrm{NAOH}$ & Hidróxido de Sódio $(\mathrm{NaOH})$ & Aspen \\
\hline
\end{tabular}

É importante notar que os fosfatídeos não estão originalmente presentes no banco de componentes do Aspen, sendo necessário informar sua estrutura molecular e suas propriedades termodinâmicas. O procedimento seguido para obtenção destas informações foi feito de forma semelhante ao adotado por Wooley e Putsche (1996), que desenvolveram um banco de dados de propriedades físicas para componentes relacionados à produção de biocombustíveis para o Aspen Plus®.

A escolha dos elementos representativos para os fosfatídeos e a sua estrutura molecular foram adotados conforme adaptações que se aproximassem dos componentes reais e pudessem ser inseridas no software. É importante notar que o Aspen Plus ${ }^{\circledR}$, apesar de permitir a inserção de moléculas contendo os átomos de fósforo e nitrogênio fazendo 5 ligações, de átomos como o cálcio (para composição dos sais de ácidos fosfatídicos NHPs), e de elementos com carga (como em íons híbridos, por exemplo), não possui modelos de contribuição de grupos capazes de estimar as propriedades de moléculas contendo estes elementos. Dessa forma, as moléculas foram adaptadas para os átomos de fósforo e nitrogênio 3, e o NHP foi inserido já em sua forma hidratável, como disposto na tabela 2.

Tabela 2 - Definição e adaptação dos fosfatídeos.

\begin{tabular}{|l|l|l|l|}
\hline Fosfatídeos & Nome do componente & Fórmula Original & Fórmula Adaptada \\
\hline Hidratável & (1,2-dilinoleoyl)-3-lecithin & C44H80NO8P & C43H78NO7P \\
\hline Não-Hidratável & DLPE-linoleoyl & C41H74NO8P & C41H74NO7P \\
\hline
\end{tabular}

Para que o software empregue os modelos de contribuição de grupos com estas moléculas, faz-se necessário ainda inserir algumas propriedades termodinâmicas. DíazTovar (2011) desenvolveu um banco de dados destas propriedades, exatamente com a finalidade de possibilitar modelagens com suporte computacional a tecnologias de processamento de lipídios. Além disso, o oleato de sódio presente no banco de dados do Aspen também precisa destas propriedades, as quais foram aproximadas para equivalentes às do ácido oleico. A tabela 3, apresenta todas as propriedades que foram inseridas manualmente na simulação. 
Tabela 3 - Parâmetros definidos manualmente para o NHP, HP e SOAPS.

\begin{tabular}{|c|c|c|c|}
\hline & \multicolumn{3}{|c|}{ Componentes } \\
\hline Parâmetro & NHP & HP & SOAPS \\
\hline Temperatura Normal de Ebulição (K) & 820,34 & - & 467,7 \\
\hline Massa Molar & 742,01 & 941,07 & 304,443 \\
\hline Temperatura Crítica (K) & 865,43 & 898 & 937,21 \\
\hline Pressão Crítica $(\mathrm{kPa})$ & 398,07 & 181,8 & 1332,96 \\
\hline Volume Crítico (m³/kmol) & 2,933 & 3,4702 & 1,05 \\
\hline Fator de Compressibilidade Crítico & 0,16227 & 0,1521 & - \\
\hline Entalpia Padrão de Combustão a $25^{\circ} \mathrm{C}(\mathrm{kJ} / \mathrm{mol})$ & $-2,7622 \times 107$ & $-1,6697 \times 107$ & - \\
\hline Temperatura de Congelamento (K) & 431,74 & 554,42 & 289,45 \\
\hline Entalpia de Formação de Gás Ideal a $25^{\circ} \mathrm{C}(\mathrm{kJ} / \mathrm{kmol})$ & $-7,6056 \times 108$ & $-7,6056 \times 108$ & -562440 \\
\hline Energia Livre de Formação de Gás Ideal a $25^{\circ} \mathrm{C}(\mathrm{kJ} / \mathrm{kmol})$ & $-1,4389 \times 107$ & $-1,4389 \times 107$ & -84840 \\
\hline Zwitterion (íon híbrido) & Sim & Não & - \\
\hline $\begin{array}{l}\text { Entalpia de Formação a Diluição Infinita em Fase Aquosa e } \\
25^{\circ} \mathrm{C}(\mathrm{kJ} / \mathrm{mol})\end{array}$ & - & - & $-764,8$ \\
\hline
\end{tabular}

O modelo termodinâmico utilizado na simulação foi o NRTL-RK. Esta decisão foi tomada ao comparar resultados obtidos por diversos modelos termodinâmicos que por vezes apresentavam valores irreais e não observados na prática. Por exemplo, seja uma mistura 96,62\% TGA e 3,38\% WATER: nas condições típicas de temperatura e pressão da neutralização $\left(80\right.$ a $100^{\circ} \mathrm{C}$ e 3 bar), o modelo Peng-Robinson indica o surgimento de uma fase vapor, a qual não acontece na prática. Deste modo, a alternativa encontrada para realizar as simulações foi o modelo NRTL-RK.

A única reação química inserida, na programação do Aspen, foi a neutralização dos ácidos graxos livres (FFA) em oleatos de sódio (SOAPS):

$$
\mathrm{R}-\mathrm{COOH}+\mathrm{NaOH} \rightarrow \mathrm{R}-\mathrm{COONa}+\mathrm{H} 2 \mathrm{O}
$$

\section{FFA NAOH SOAPS WATER}

A elaboração dos flowsheets da neutralização, está representada na Figura 2. A principal dificuldade encontrada neste ponto foi com relação à modelagem das centrífugas - o modelo de centrífugas presente no Aspen Plus ${ }^{\circledR}$ consiste apenas em separações contendo partículas em estado sólido. O que acontece na realidade é que a borra (fase pesada da neutralização) na verdade tendem a se comportar como emulsões, sendo, portanto, aproximadas para fases líquidas. Dessa forma, foi necessário utilizar outro bloco para realizar esta separação.

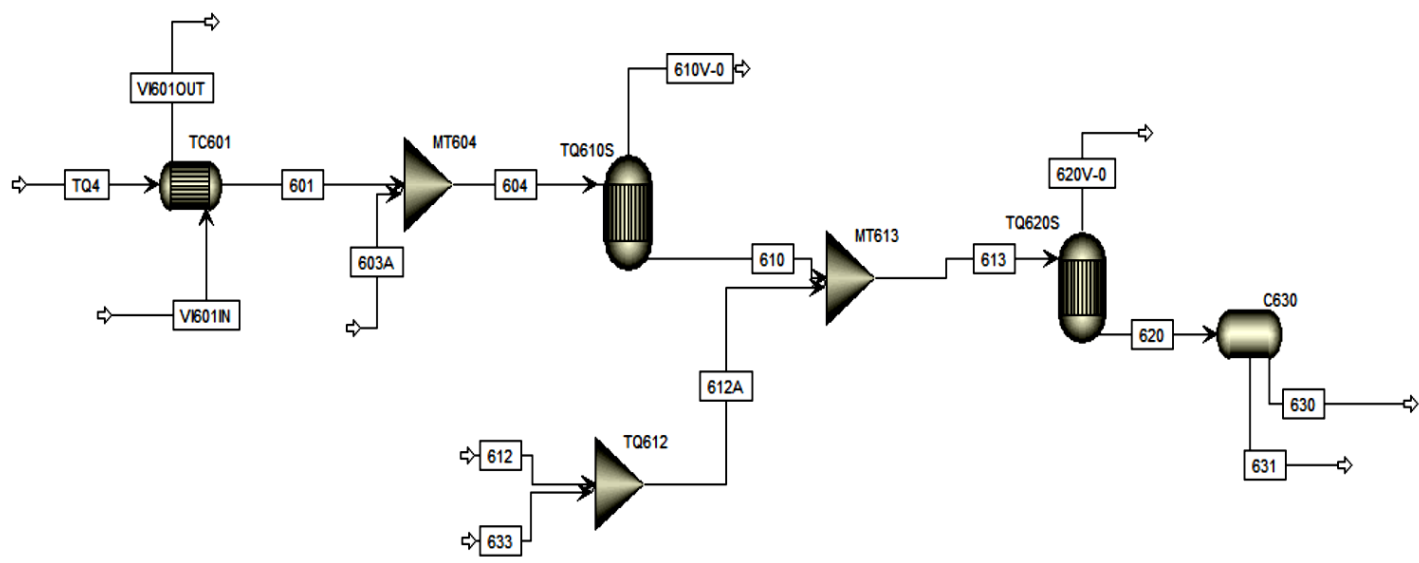

Figura 2 - Flowsheet do modelo da neutralização elaborado no Aspen Plus ${ }^{\circledR}$ 
O bloco escolhido foi o "DECANTER", o qual atua como separador de 2 fases líquidas. A forma como está separação ocorre pode ser controlada através de um coeficiente de distribuição líquido-líquido (constante de equilíbrio) KLL:

$x_{2,1}=E_{i} \cdot K_{L L, i} \cdot x_{1}$

- $x_{n, 1}$ é a fração mássica do componente "i” na fase "n".

- $E_{i}$ é a eficiência de separação (será admitida como 1).

Esta constante de equilíbrio pode ser avaliada por uma correlação incorporada no software em função da temperatura:

$$
\operatorname{Ln} K_{L L}=a+\frac{b}{T+c} \cdot \operatorname{LnT}+d \cdot T
$$

\section{Resultados e Discussões}

No trabalho realizado por VIEIRA FILHO (2012) na refinaria da cooperativa Cocamar ${ }^{\circledR}$, foram realizados ensaios na refinaria, nos quais foram avaliadas as composições mássicas do óleo neutralizado e da borra (ou seja, valores de x1,i e x2,i). Utilizando um destes conjuntos de valores, junto da ferramenta SOLVER do Microsoft Excel®, foi possível determinar parâmetros a, b, c e d capazes de fazer uma separação semelhante à da centrífuga.

Deste modo os valores obtidos foram plenamente satisfatórios. A figura a seguir apresenta uma captura de tela do software com os parâmetros a, b, c e d utilizados para a correlação de cada um dos componentes na centrífuga da neutralização.

\begin{tabular}{|c|c|c|c|c|c|c|}
\hline $\begin{array}{l}\text { Coe } \\
\text { Basi }\end{array}$ & is Mass & $\ln (\mathrm{KLL}$ & )$=a+b / T+c \ln ($ & ) $+d T$ & & \\
\hline & & & & & & \\
\hline b & Coefficient & TGA & WATER & FFA & NHP & HP \\
\hline z & a: & -0.0001051 & 1.19814 & 0.914535 & 0.80597 & 6.997 \\
\hline s & b: & 0.000567883 & 1.00222 & 0.998332 & 0.997742 & 6.37214 \\
\hline b & c: & -0.000475759 & 1.16639 & 0.415225 & 0.289919 & 1.05952 \\
\hline$b^{2}$ & d: & -0.00248101 & -0.0129502 & -0.00451117 & -0.1 & -0.0021633 \\
\hline
\end{tabular}

Figura 3 - Parâmetros inseridos na correlação para cálculo do $K_{L L}$ de cada componente na centrífuga da neutralização

Contudo, é importante notar que os parâmetros da correlação foram ajustados para um único ponto experimental. Isto significa que a influência da temperatura talvez não esteja plenamente condizente com o real. Uma análise de sensibilidade avaliando a fração mássica de TGA na corrente de borra em diferentes temperaturas. 


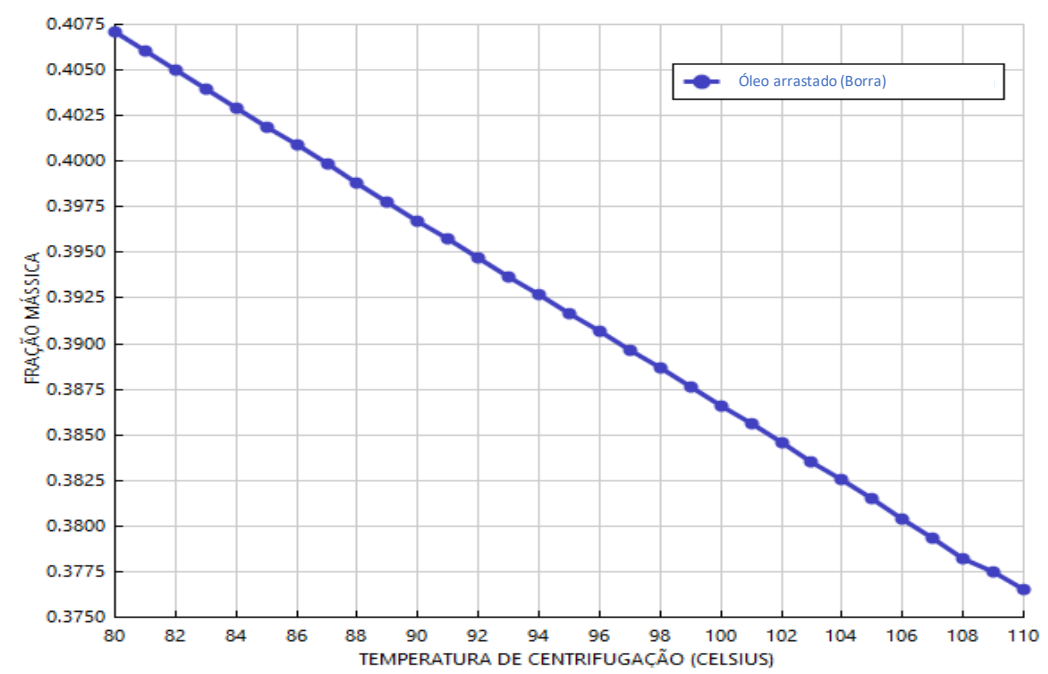

Figura 4 - Arraste de óleo em função da temperatura para a correlação empregada

Dessa forma, resultados melhores podem ser obtidos aumentando os pontos de análises de separação em diferentes temperaturas, formando um conjunto de dados ( $\mathrm{T}$, $K_{L L}$ ) e portanto, permitindo um ajuste mais rigoroso dos parâmetros a, b, c e d de cada componente.

\section{Conclusão}

A modelagem e simulação de processos industriais para os cenários brasileiros vem ganhando espaço, mas ainda há muito o que ser explorado. Portanto este trabalho buscou criar oportunidades por meio da criação de parâmetros que se correlacionassem com a prática do processo de refino de óleo de soja na etapa de neutralização.

Foi possível concluir que o modelo proposto se próxima dos fatores e parâmetros industriais da etapa de neutralização e que podem serem mais explorados criando novas faixas de simulação com variações da temperatura e dos parâmetros industriais.

\section{Referencias}

1. ANDERSON, D. Edible Oil and Fat Products Processing Technology, Bailey's Industrial Oil and Fat Products. vol. 4, ed. Wiley-Interscience Publication, John Wiley \& Sons, Cap. 1, A Primer on Oils Processing Technology, p. 1-60b, 1996.

2. DE PRETTO, C.; TARDIOLI, P. W.; COSTA, C. B. B. Assessing energetic and available fuel demands from a soybean biorefinery producing refined oil, biodiesel, defatted meal and power. Computers and Chemical Engineering, 104, 259-270, 2017.

3. DÍAZ-TOVAR, C. A.; GANI, R.; SARUP, B. Computer-Aided Modelling of Lipid Processing Technology. Ph.D. Thesis. Department of Chemical and Biochemical Enginerring, Technical University of Denmark, 2011.

4. FRÉ, Nicéia Chies da. Obtenção de ácidos graxos a partir da acidulação de borra de neutralização de óleo de soja. 2009. 111 f. Dissertação (Mestrado) Departamento de Engenharia Química, Universidade Estadual do Rio Grande do Sul, Porto Alegre, 2009. 
5. GRANJO, J.; DUARTE, B.; OLIVEIRA, N. Soybean Biorefinery: Process Simulation and Analysis. Chemical Engineering Transactions, vol. 45, 2015.

6. HAMMOND, Earl G.; JOHNSON, L. A.; SU, C.; WANG, T.; and WHITE, P. J.; Bailey's Industrial Oil and Fat Products: Edible Oil and Fat Products: Edible Oils. 6. ed. Ames, Iowa: Wiley-Interscience, Cap. 13, p. 577-653. Vol 2. Iowa State University. 2005 .

7. LANDUCCI, G.; PANNOCCHIA G.; NICOLELlA, C.; PELAGAGGE, L. Analysis and simulation of an industrial vegetable oil refining process. Journal of Food Engineering, 2013, 840-851.

8. VIEIRA FILHO, F. C. Modelagem e Análise do Processo de Neutralização no Refino de Óleo de Soja. Dissertação de Mestrado. Departamento de Engenharia Química - Centro de Tecnologia. Universidade Estadual de Maringá, 28 de junho de 2012, Maringá, Paraná, Brasil.

9. WOOLEY, Robert J.; PUTSCHE, Victoria. Development of an ASPEN PLUS Physical Property Database for Biofuels Components. Colorado: Nrel, 1966. 36 p. Disponível em: 〈http://infohouse.p2ric.org/ref/22/21210.pdf>. Acesso em: 05 dez. 2017. 